This document is confidential and is proprietary to the American Chemical Society and its authors. Do not copy or disclose without written permission. If you have received this item in error, notify the sender and delete all copies.

\title{
Timoshenko Bending and Eshelby Twisting Predicted in Molecular Nanocrystals
}

\begin{tabular}{|r|l|}
\hline Journal: & The Journal of Physical Chemistry \\
\hline Manuscript ID & jp-2018-08261s.R1 \\
\hline Manuscript Type: & Article \\
\hline Dute Submitted by the & n/a \\
\hline Complete List of Authors: & $\begin{array}{l}\text { Li, Chao; New York University, Chemistry and Molecular Design Institute } \\
\text { Shtukenberg, Alexander; New York University, Chemistry and Molecular } \\
\text { Design Institute } \\
\text { Carter, Damien; Curtin University, Nanochemistry Research Institute } \\
\text { Cui, Xiaoyan; East China Normal University, Chemistry } \\
\text { Olson, Isabel; New York University, Chemistry and Molecular Design } \\
\text { Institute } \\
\text { Rohl, Andrew; Curtin University, Chemistry } \\
\text { Gale, Julian; Curtin University, Nanochemistry Research Institute, } \\
\text { Department of Chemistry } \\
\text { Raiteri, Paolo; Curtin University, Department of Chemistry } \\
\text { Kahr, Bart; New York University, Chemistry and Molecular Design } \\
\text { Institute }\end{array}$ \\
\hline
\end{tabular}




\title{
Timoshenko Bending and Eshelby Twisting Predicted in Molecular Nanocrystals
}

Chao Li, ${ }^{\dagger}$ Alexander G. Shtukenberg, ${ }^{\dagger}$ Damien J. Carter ${ }^{\star}$ Xiaoyan Cui ${ }^{\dagger}$ Isabel Olson,$^{\dagger}$ Andrew L. Rohl, Julian D. Gale, $\stackrel{\ddagger}{\ddagger}$ Paolo Raiteri, $* \vdots$ and Bart Kahr $* \dagger$

†Department of Chemistry and Molecular Design Institute, New York University, New York City, New York 10003, United States

Curtin Institute for Computation and School of Molecular and Life Sciences, Curtin University, P.O. Box U1987, Perth, Western Australia 6845, Australia

\section{Corresponding Author}

*Email: bart.kahr@nyu.edu,p.raiteri@curtin.edu.au

\begin{abstract}
Well-formed crystals are polyhedral with flat facets and sharp edges. Nevertheless, a remarkable number of molecular crystals can bend and twist during growth. Many others can be distorted by applying external forces or creating heterogeneities by thermal or photochemical reaction. As part of an effort to identify the forces that so commonly deform molecular crystals and to characterize their consequences, a force field is evaluated for its ability to predict mechanical distortions in nanocrystals. Macroscopic materials provide estimates of the expected responses that were tested here in silico for "molecular bimetallic strips" created from rods of iodoform and bromoform in smooth contact, and nanocrystalline rods of iodoform with left and right screw dislocations. It was demonstrated that an optimized force field based largely on Amber parameters matches expectations for elastic and plastic distortions, despite the fact that these mechanical responses are far removed from the force field parametrization set.
\end{abstract}

\section{TOC GRAPHICS}

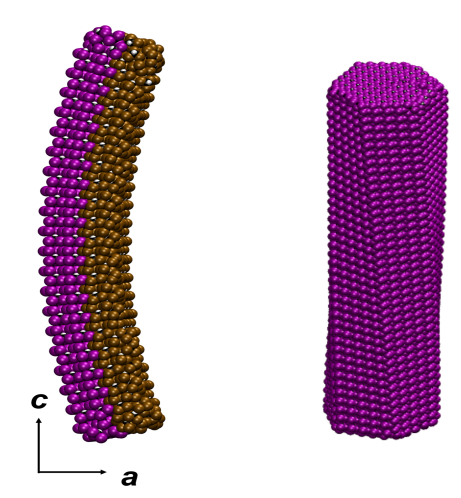

KEYWORDS. Bimetallic strip, Eshelby twist, nanocrystal, screw dislocation, strain, stress 
Introduction. We have been investigating the common phenomenon of crystals that twist as they grow. ${ }^{1,2,3}$ These molecular crystals include the following, among many others: hippuric acid, ${ }^{4}$ resorcinol, ${ }^{5}$ testosterone propionate, ${ }^{6}$ mannitol, ${ }^{7}$ aspirin, ${ }^{8}$ malic acid, ${ }^{9}$ and DDT. ${ }^{10}$ In addition to studies of growth-induced crystal twisting, a subject that finds its progenitors in earlier work, there has been a renaissance ${ }^{11}$ in the study of molecular crystals that respond mechanically to external stimuli. ${ }^{12}$ This new focus is grounded in research from the $1980 \mathrm{~s}^{13,14,15}$ but has become an explosive frontier in materials science driven by the new recognition that molecular crystals are neither stiff nor brittle, but rather elastic and plastic. In particular, photoirradiation, ${ }^{16,17,18}$ and applied stresses ${ }^{19,20,21,22,23,24}$ can affect dramatic reversible and/or irreversible changes of shape. Therefore, tools for the atomistic simulation of mechanical deformations as a consequence of chemistry or applied forces will become more important as emphases evolve from the characterization of bending and twisting to establishing mechanism. Predicting the response is requisite if we aim to transduce energy of photons, for example, to predetermined mechanical motion or work. ${ }^{25}$ Moreover, evidence has begun to accumulate that nano-scale molecular crystals do not necessarily express the translational symmetry of macrocrystals. ${ }^{26,27}$ Morphology may evolve along the way. Tools for direct observation, and tools for modeling observations need to evolve in tandem.

For these reasons, we needed an efficient computational tool for the evaluation of the mechanical deformations of molecular crystals. Atomistic simulations using force fields offer the possibility of investigating bulk deformations of crystal structures in terms of the configurations of molecules in the solid state. Here, we investigate to what extent empirical force fields are able to predict the bending and twisting of nanoscale molecular crystals.

Parametrized force fields have changed the practice of chemistry by arming researchers with powerful tools for predicting structure and energy, and consequently quickly evaluating competing hypotheses. One of the most successful implementations of the so-call molecular mechanics method, the AMBER (Assisted Model Building with Energy Refinement) force field, was developed primarily for the investigation of biomolecular structures with innumerable applications to proteins ${ }^{28}$ and nucleic acids. ${ }^{29}$ GAFF (General Amber Force Field) was subsequently introduced to handle organic compounds more generally and it was shown to well 
reproduce crystallographic structures. ${ }^{30,31}$ Indeed, one of the goals of force field developers is the creation of tools that can be broadly transferable to chemical systems that lie outside of the set of molecules for which it was parametrized. GAFF, for instance, recently was optimized for predicting transition temperatures in thermotropic liquid crystals, ${ }^{32}$ an application far from the target of the original AMBER parameterization. Here, we attempt to extend an optimized force field to the prediction of the elastic and plastic deformation of molecular crystals.

The molecular crystalline materials investigated herein are iodoform $\left(\mathrm{CHI}_{3}\right)$ and bromoform $\left(\mathrm{CHBr}_{3}\right)$. They were chosen because they are small, penta-atomic molecules, with no torsional degrees of freedom and their crystal structures (see Figure 1A for iodoform) are isomorphous. ${ }^{33}$ There has been some ambiguity concerning the room temperature crystal structure of iodoform. The first X-ray diffraction studies suggested the polar, enantiomorphous space group, $P 6_{3},{ }^{34,35}$ but later neutron scattering data was more consistent with the non-polar, achiral space group $P 6_{3} / m \cdot{ }^{36,37}$ The latter symmetry was accommodated by disorder in which the large iodine atoms were in identical positions, but the $\mathrm{C}-\mathrm{H}$ bonds along the hexagonal axis were distributed in both the $\pm c$ directions. We regularized the crystallographic information file (.cif) for the neutron structure so as to eliminate the superposition of molecules that appears in the average unit cell by virtue of disorder but that could not be accommodated in the calculations (Table S4). The bromoform crystal structure was likewise purged of the disorder and reflection symmetry (Table S5). Crystal parameters in the non-disordered structures were the following: iodoform, $a=7.036 \AA$, c=7.315 ̊; bromoform, $a=6.437 \AA$, $c=6.873 \AA$.

Bending. Two strips of different metals in smooth contact will bend as temperature changes as a function of their differential thermal expansion. This behavior is the basis of thermostats and has been thoroughly investigated by Timoshenko. ${ }^{38}$ The bending of a bimetallic strip is given by equation (1):

$$
\kappa=\frac{6 \varepsilon(1+m)^{2}}{3(1+m)^{2}+(1+m n)\left(m^{2}+\frac{1}{m n}\right)} \cdot \frac{1}{h}
$$

Here, $\kappa$ is the curvature. Misfit strain $\varepsilon$ at the interface can be obtained from the difference between the thermal expansion coefficients of the two components $(\Delta \alpha)$ and the temperature change $(\Delta T), \varepsilon=\Delta \alpha \Delta T$. Alternatively, a static stress can be induced by structural differences. Here, we explore the strain at the interface of isomorphous iodoform and bromoform, a "molecular bimetallic strip" so to speak. The misfit strain $\varepsilon=\left(a_{\mathrm{I}}-a_{\mathrm{Br}}\right) / a_{\mathrm{I}}$ where $a_{\mathrm{I}}$ and $a_{\mathrm{Br}}$ are 
the lattice constants in the plane of the interface for the direction of bending, of iodoform and bromoform, respectively. $h$ is the total thickness of the bilayer, $m$ is the ratio of thickness of the two components, and $n$ is the ratio of Young's moduli (see methods section). Recently molecular crystalline analogues of bimetallic strips, twinned crystals of 1,2,4,5-tetrabromobenzene, ${ }^{39}$ were found to behave like metals when heated, where the elastic deformation is driven by misfit strain at the interface.

First, we considered the (10 $\overline{1} 0)$ interface constructed in silico from finite rods of iodoform measuring $4 \times 4 \times 21$ unit cells and increasing in thickness to $6 \times 4 \times 21,8 \times 4 \times 21,10 \times 4 \times 21$ and $12 \times 4 \times 21$ unit cells. In other words, the bilayers were 21 cells extended along the $c$ axis, 4 cells deep along $b^{*}$, with a height along $a$, perpendicular to the iodoform/bromoform interface varying from 4-12 unit cells in increments of two. The iodine atoms on one side of the strip were re-labelled as bromine atoms, effectively placing a crystallite of iodoform in epitaxial contact with a crystallite of bromoform, but with initially exaggerated lattice constants and bond lengths for the latter as it was derived from the iodoform crystal structure. The structure was allowed to evolve at a temperature of $50 \mathrm{~K}$ producing a misfit strain at the interface. The bilayer spontaneously bent as shown in Figure 1B. The degree of bending was quantified as the reciprocal of the radius of the best fit circle to the carbon atoms in the central row of the interfacial layer of iodoform and bromoform. The predictions of Timoshenko can be calculated from eq(1) with the $c$-lattice constants from the optimized, non-disordered structures where $c_{\mathrm{I}}=$ $7.315 \AA, c_{\mathrm{Br}}=6.873 \AA$. Since the rods are extended along $c$, and bending is evaluated only along the length of the rods, only the $c$-lattice constant mismatch was included in eq(1). The results matched the predictions of Timoshenko with fidelity (Figure 1C). As to be expected, the slenderer the strip, the larger the deviation from Timoshenko's prediction for bulk materials, undoubtedly due to surface relaxation leading to local changes in the elastic properties, such that the rods are no longer elastically homogeneous. Such effects will diminish as the ratio of surface and bulk contributions. Care is needed to treat the electrostatic interactions by direct summation, as appropriate to a finite system due to the overall dipole moment created as a result of bending. See Methods section. 
A
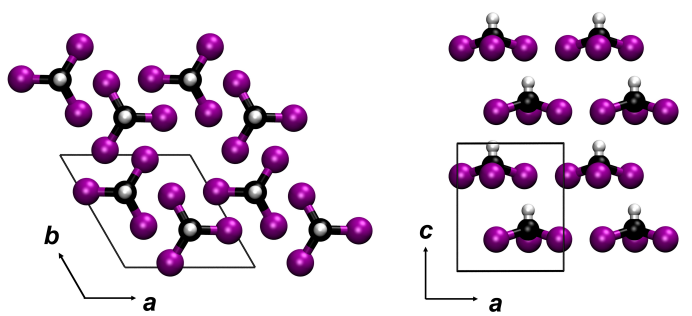

B
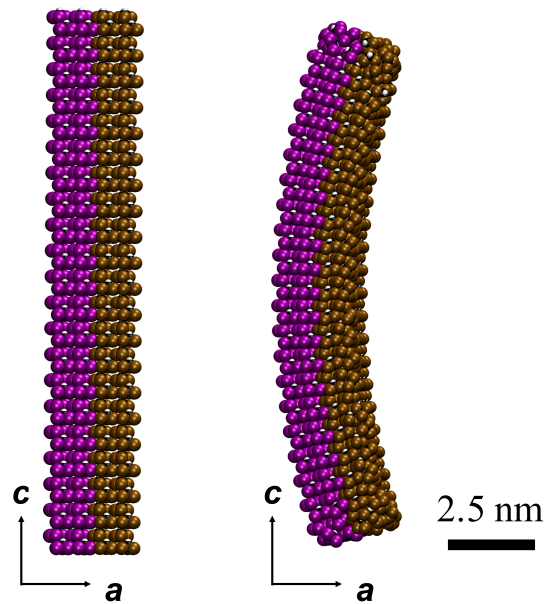

C

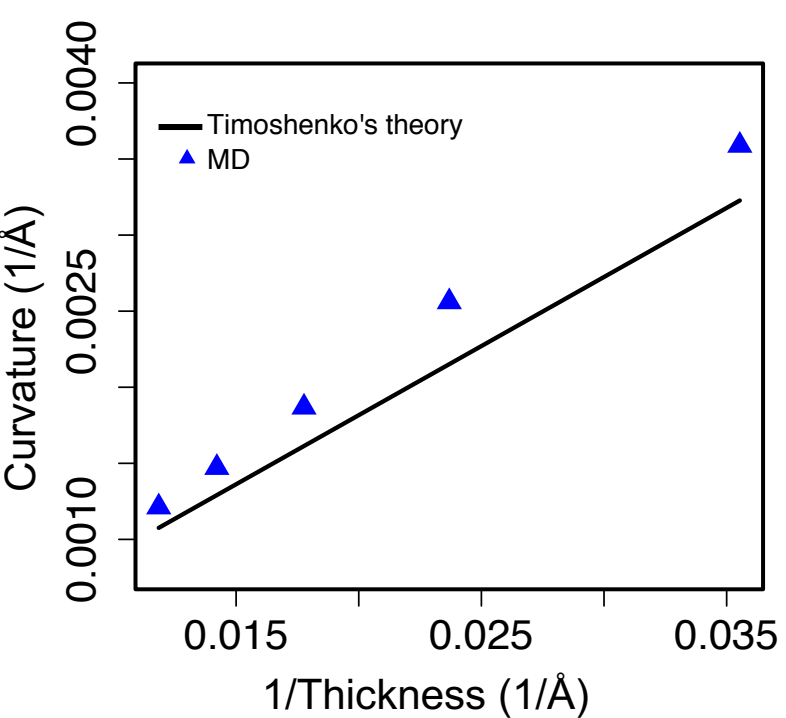

Figure 1. Molecular bilayers consisting of iodoform and bromoform; (1010) strained interface. (A) Iodoform crystal structure viewed along $b$ and $c$. (B) Half of the iodine atoms (purple) on one side of the iodoform nanocrystal were redefined as bromine (brown). The misfit strain within the $a c$ plane produced a pronounced bending of the crystalline bilayer after relaxation at $50 \mathrm{~K}$ during MD. (C) Comparison of MD computed curvature for six rods of differing thickness (4, 6, 8,10 , and 12 unit cells thick in the $a$ direction, blue triangles) with the prediction of Timoshenko (black line). ${ }^{38}$ 
We sought to establish whether there would be any differences found in bending toward or away from the polar axis. Such structures would be symmetry inequivalent bilayers. In other words, by stacking strips along [0001] first with iodoform "on top" with greater $c$ lattice constants, and then with bromoform on top, we would produce inequivalent structures bending away from or towards $+c$, respectively. Directional bending in polar thin films was observed for $\mathrm{InSb}, \mathrm{GaAs}$, InAs, GaSb, AlSb crystals ${ }^{40}$ and simulated using MD on SiGe as a consequence of the differential surface tension on the $\pm c$ planes. ${ }^{41,42}$

The interfacial strain induced in the bimetallic strip with a (0001) interface was too large and during the MD simulations the appearance of one (or more) edge dislocations allowed for a larger reduction in energy than the bending of the strip itself. The slippage of one crystallite with respect to the other (Figure 2A), where $n$ molecules of bromoform were only in contact with $n-1$ molecules of iodoform, caused a reduction of the bending of the bimetallic strip. A step was produced at the (0001) terrace with an iodoform molecule extending from the rod but not in contact with the underlying bromoform. We did not see this collapse when the polar axis was

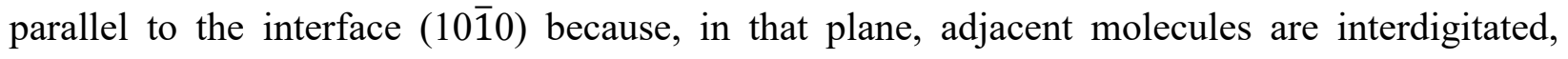
whereas the (0001) plane is smooth and can accommodate sliding without a large activation energy (Figure S1). In order to avoid the collapse of the bromoform half-rod, we repeated the simulation but in a bimolecular strip in which the short side contained alternating bromoform and iodoform molecules, mitigating the strain (Figure $2 \mathrm{~B}$ and $2 \mathrm{C}$ ). In this case, the misfit strain is given by $\varepsilon=\left(a_{\mathrm{I}}-a_{\mathrm{avg}}\right) / a_{\mathrm{I}}$ where $a_{\mathrm{I}}=7.035 \AA, a_{\mathrm{avg}}=\left(a_{\mathrm{I}}+a_{\mathrm{Br}}\right) / 2=6.736 \AA$. Once again, we found that the radii of curvature deviated from the computed values of Timoshenko and from one another at only very small thicknesses $(31 \times 4 \times 4$ unit cells). As the rod became thicker, the structure began to converge to the bulk material prediction (Figure 2D) irrespective of whether iodoform or bromoform was on top. In summary, the force field performed exceptionally well in predicting the bending of iodoform/bromoform bilayers and the bending was indifferent to the direction of the polar axis. 
Figure 2. Bilayers with heterogeneous interfaces normal to polar axis; (0001) strained interface. (A) A simulation with a strip constructed from $31 \times 4 \times 10$ unit cells, but with all the iodine atoms on the top being redefined as bromines. This construction was unstable at the interface and only bent modestly. (B) Every other iodine atom on the top half of the iodoform crystal $(31 \times 4 \times 4)$ was redefined as bromine. The strip showed upward bending toward the $+c$ direction after relaxation during MD. (C) By switching the order of layers, the strip bent downward toward the $-c$ direction. (D) MD computed curvature of bending upward (red squares) and bending downward (blue circles) compared with the prediction of Timoshenko (black line). ${ }^{38}$ 
Table 1 reports the difference in energy between the bilayer and the optimized isolated layers at infinite separation; in other words, this is includes the strain energy to bring the crystals to the same lattice spacing at the interface, the gain in energy upon assembling the two halves into a bilayer and the strain relief brought by the bending of the rod. The data show a smaller per molecule relaxation as the rods get thicker, which is consistent with the notion that it is impossible to make a straight infinitely thick epitaxial bilayer without the inclusion of other stress relieving mechanisms, such as dislocations.

Table 1. Potential energies before and after bending

\begin{tabular}{|c|c|c|}
\hline Size & $\begin{array}{c}\text { Energy Difference } \\
\text { per molecule } \\
(\mathrm{kJ} / \mathrm{mol})\end{array}$ & $\begin{array}{c}\text { Energy } \\
\text { difference/Area } \\
\left(\mathrm{J} / \mathrm{m}^{2}\right)\end{array}$ \\
\hline $2 \times 4 \times 21$ & -5.93 & -0.153 \\
\hline $3 \times 4 \times 21$ & -3.92 & -0.152 \\
\hline $4 \times 4 \times 21$ & -2.89 & -0.149 \\
\hline $5 \times 4 \times 21$ & -2.28 & -0.147 \\
\hline $6 \times 4 \times 21$ & -1.87 & -0.145 \\
\hline
\end{tabular}

Twisting. Real crystals are riddled with dislocations. Axial screw dislocations in wirelike crystals can twist the wire around the direction of the Burgers vector, ${ }^{43,44}$ as established by Eshelby. ${ }^{45,46}$ The twist angle $\theta=\pi / P=k \vec{b} / A$, where $P$ is the pitch $\left(180^{\circ}\right.$ rotation of the wire around its axis), $\vec{b}$ is the magnitude of the Burgers vector, $A$ is the cross-sectional area, and $k$ is a geometrical constant. For a regular hexagonal cross section, $k=1.015 .{ }^{46}$ Spectacular Eshelby twisted nanocrystals of lead sulfide, ${ }^{47}$ lead selenide, ${ }^{48}$ and silicon ${ }^{49}$ were recently imaged. Hence, to produce a helical elastic deformation, an axial screw dislocation with a Burgers vector parallel to the $c$ axis was imposed in hexagonal cylinders of iodoform. Perfect hexagonal iodoform nanocrystals 30 unit cells long, with thicknesses of the hexagonal faces ranging from 4-8 unit cells in increments of one cell were constructed. To generate a right-handed screw dislocation with Burgers vector $\vec{b}=c<0001>$, the displacements of molecules were increased in the $c$ direction uniformly from zero to $\vec{b}$ as the vector from the center of the cylinder and the molecule rotating through $2 \pi$ with $<10 \overline{1} 0>$ as the reference vector (Figure $4 \mathrm{~A}$ ). In other words, the displacement vector $\mu=\frac{\vec{b}}{2 \pi} \tan ^{-1} \frac{y}{x}$. For a left-handed screw dislocation, the molecules were displaced in the $c$-direction from zero to $-\vec{b}$. The shear direction of [2 $\overline{1} \overline{1} 0]$ is compared in the 
supporting material (Figure S3) with the shear direction of [1010] illustrated below. The structures, energies and twisting angles were indifferent to the choice of shear planes parallel to the $c$ axis.
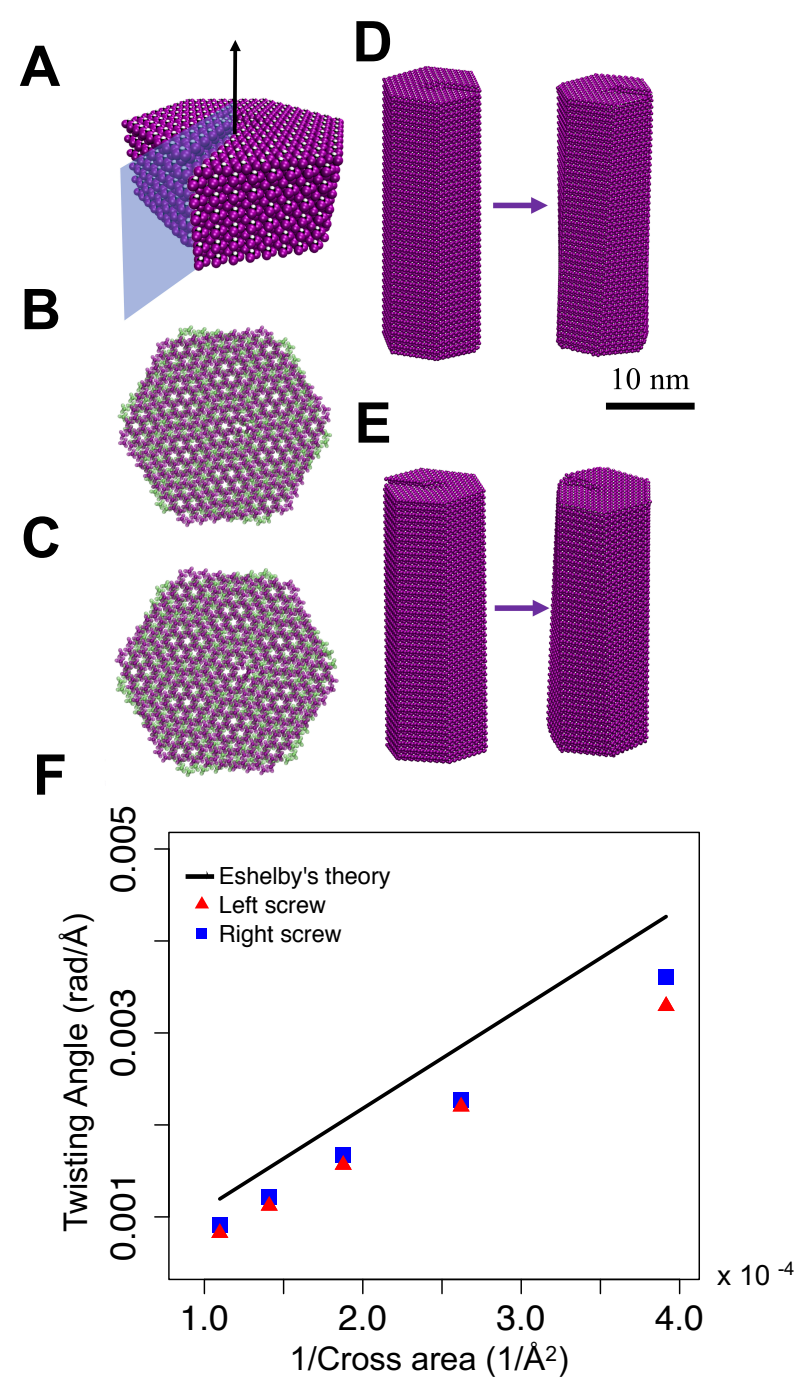

Figure 3. Twisted iodoform nanocrystals with diastereomorphous screw dislocations (Burgers vector $\vec{b}=c<0001>$ ). (A) Illustration of the generation of a right-handed screw dislocation in a short hexagonal iodoform cylinder measuring eight unit cells on a side and six unit cells in length $(8 \times 6)$. (D,E) Longer hexagonal iodoform nanocrystals $(8 \times 30)$ with a left-handed screw dislocation (D) and a right-handed screw dislocation (E) showed Eshelby twist after MD simulations. (B,C) The twist is evident by overlapping the top layer (purple) and the bottom layer 
(green) in the nanocrystal. (F) Comparison of MD simulated twisting angle for both left(triangles) and right-handed (squares) screw dislocations with the prediction of Eshelby. ${ }^{45,46}$

After MD relaxation, the ensemble of hexagonal nanocrystalline rods with both left-handed and right-handed screw dislocations, underwent an Eshelby twist (Figure 3D, 3E). By investigating the twist intensity, which is shown in Figure 3F, our simulations present good agreement with the model of Eshelby. The greatest deviations were found in the slenderest rods, with pitch conforming to the theory of Eshelby as the rods became thicker.

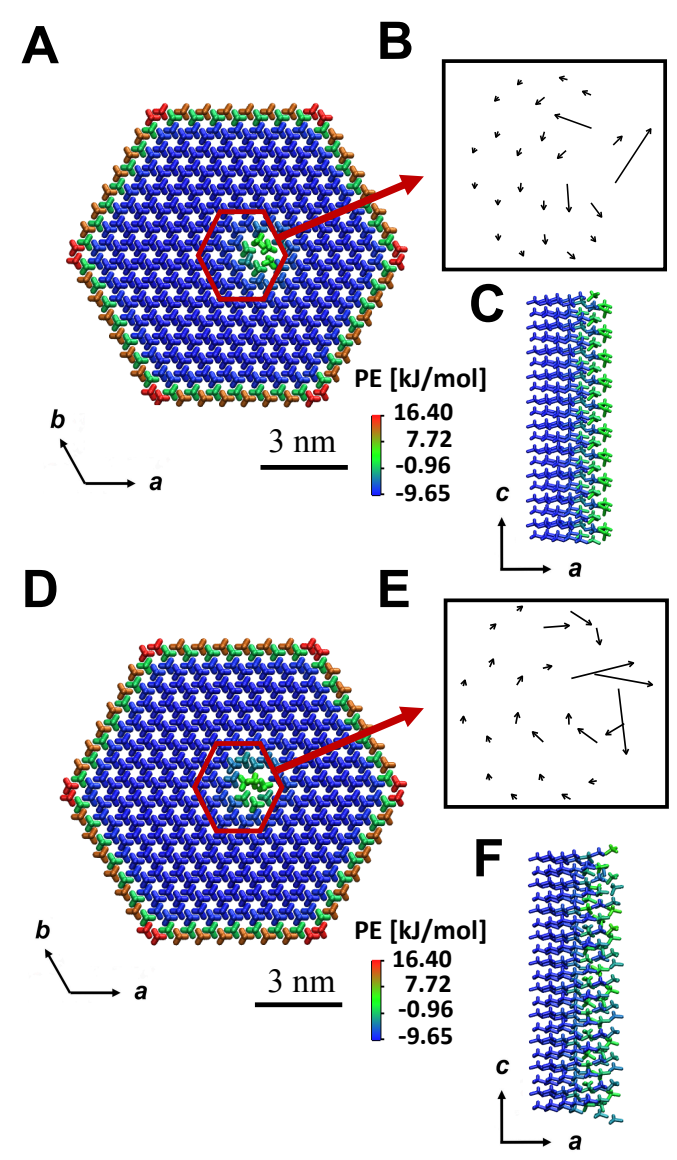

Figure 4. Structures and energies of screw dislocations in hexagonal iodoform nanocrystals. (A,D) Potential energy is plotted by color for molecules in for both left-handed screw dislocated (A) and right-handed screw dislocated structures. (D) Cores have high energies. The red hexagonal boxes highlighted the original core location. (B,E) showed reorientation of the molecules in the core. The vectors are drawn from carbon to hydrogen in one iodoform molecule 
and their lengths are scaled by a factor of 10. (C,F) Left-handed screw (C) and right-handed screw dislocation $(\mathrm{F})$ viewed along $b$.

The smallest, most twisted rods showed the greatest relaxation as they are mostly core, with little corona. In other words, the plastic deformation overwhelms the average elastic potential energy changes in the material surrounding the cores. Right- and left Eshelby twisted crystal structures of a given chiral space group are diastereomorphous and need not have the same structure or energy and it was found that the left Eshelby twisted crystal was always more stable that its right-handed counterpart.

Simulations of screw dislocations in molecular crystals provide an opportunity to illustrate the atomistic structure of the cores. ${ }^{50,51,52}$ Our simulations show that, naturally, the molecules on the surfaces have characteristically high energies because they are not fully coordinated, and the molecules in the cores likewise suffer increases in potential energy (Figure 4A, 4D). The orientation of iodoform molecules sitting in the core can be visualized as the projection of the vector from carbon to hydrogen on (0001) plane (Figure 4B, 4E). The vector field of the core showed an overall counterclockwise rotation of the molecules for left screw dislocation (Figure 4B) and a clockwise rotation for right screw dislocation (Figure 4E). The circulation, motion of molecules around the Burgers vector as indicated by the projection of the $\mathrm{C}-\mathrm{H}$ vector in the (0001) plane, is larger for molecules with higher potential energy (Figure 4C, 4F).

Since crystalline iodoform is chiral in the space group $P 6_{3}$, structural and energetic differences are expected for right-handed and left-handed screw dislocations in any one enantiomorph. ${ }^{53}$ Atomic force microscopy of dislocation spirals on $\{0001\} L$-cystine faces ${ }^{54}$ has shown a strong preference for left-handed screw dislocations. The (0110) surface of a steroid crystal $^{55}$ has also shown that screw dislocations of one sign are more energetically favorable, though the preferred sense was not indicated. As an equal number of heterochiral screw dislocations are to be expected in a bulk crystal on the basis of topological arguments, the failure to observe heterochiral spiral hillocks is likely a consequence of the differential kinetics of propagation. 
Summary. We have demonstrated that MD simulations predict the deformations of nanoscale molecular crystals such as bending by misfit strain or twisting by screw dislocations that agree with well-known predictions of the theory of elasticity. The findings are encouraging for the use of widely available force fields in the investigation of the mechanical properties of molecular crystals as a consequence of responses to photochemical or external stimuli. There appears to be no end in sight of demonstrations of photomechanical ${ }^{56,57}$ and thermomechanical crystals the bend and twist. ${ }^{58,59}$ Kinetic models have been derived to account for the heterogeneous composition that accompanies the non-uniform irradiation. ${ }^{60}$ Macroscopic models of this kind have been connected to observed bending in slender rods. ${ }^{61}$ However, a connection between macro-responses and microscopic models can be advanced, in our view, with computations of the kind described herein.

Methods. Optimizations and MD simulations were performed with the LAMMPS (Large-scale Atomic/Molecular Massively Parallel Simulator) code. ${ }^{62}$ The force field was built with the molecular parameters from GAFF. Table S1 gives the parameters for bromoform and iodoform. Only the pair potential values for $\mathrm{Br}$ and I are not the default values. The potential parameters of I and Br were fitted to reproduce the crystal structure of iodoform and bromoform which were not well fit with the default values. The partial charges were derived from quantum mechanical calculations by optimizing the molecules in NWCHEM ${ }^{63}$ using RHF with a $6-31 G^{* *}$ basis. (Table S2). Table S2 gives the partial atomic charges for $\mathrm{C}, \mathrm{H}, \mathrm{I}$, and $\mathrm{Br}$. Table 2 shows the predicted the lattice parameters for iodoform and bromoform. Young's moduli were calculated using the General Utility Lattice Program (GULP) code. ${ }^{64}$ For iodoform: $Y_{x, y}=4.8, Y_{z}=8.7 \mathrm{GPa}$; for bromoform: $Y_{x, y}=12.8, Y_{z}=7.9 \mathrm{GPa}$.

Table 2. Lattice parameters comparisons between experimental crystal structure and calculated crystals structure using modified GAFF.

Iodoform

\begin{tabular}{|l|l|l|l|l|}
\hline & Experiment $(\mathrm{RT})^{37}$ & $\begin{array}{l}\text { DFT-optimized, } \\
\text { disorder free }\end{array}$ & $298 \mathrm{~K}$ & $50 \mathrm{~K}$ \\
\hline $\mathrm{a}(\AA)$ & $6.811(2)$ & 7.035 & 7.094 & 7.047 \\
\hline $\mathrm{b}(\AA)$ & $6.811(2)$ & 7.035 & 7.094 & 7.047 \\
\hline $\mathrm{c}(\AA)$ & $7.553(2)$ & 7.316 & 7.316 & 7.344 \\
\hline$\alpha\left(^{\circ}\right)$ & 90 & 90 & 90 & 90 \\
\hline$\beta\left(^{\circ}\right)$ & 90 & 90 & 90 & 90 \\
\hline $\mathrm{V}\left({ }^{\circ}\right)$ & 120 & 120 & 120 & 120 \\
\hline $\mathrm{V}\left(\AA^{3}\right)$ & $303.44(15)$ & 313.569 & 325.431 & 315.843 \\
\hline
\end{tabular}




\section{Bromoform}

\begin{tabular}{|l|l|l|l|l|}
\hline & Experiment $(273 \mathrm{~K})^{33}$ & $\begin{array}{l}\text { DFT-optimized, } \\
\text { disorder free }\end{array}$ & $298 \mathrm{~K}$ & $50 \mathrm{~K}$ \\
\hline $\mathrm{a}(\AA)$ & $6.333(17)$ & 6.437 & 6.484 & 6.446 \\
\hline $\mathrm{b}(\AA)$ & $6.333(17)$ & 6.437 & 6.484 & 6.446 \\
\hline $\mathrm{c}(\AA)$ & $7.226(3)$ & 6.873 & 7.002 & 6.899 \\
\hline$\alpha\left(^{\circ}\right)$ & 90 & 90 & 90 & 90 \\
\hline$\beta\left(^{\circ}\right)$ & 90 & 90 & 90 & 90 \\
\hline $\mathrm{Y}\left({ }^{\circ}\right)$ & 120 & 120 & 120 & 120 \\
\hline $\mathrm{V}\left(\AA^{3}\right)$ & 250.97 & 246.645 & 254.940 & 248.255 \\
\hline
\end{tabular}

All simulations were performed in the NVT ensemble for at least $10 \mathrm{~ns}$ with a $1 \mathrm{fs}$ timestep. Typically, MD simulations employ periodic boundary conditions and the Ewald summation method or truncated real space methods, such as the Wolf summation with damping shift force (DSF), to treat the long-range electrostatic interactions. However, it is important to stress that neither approach is appropriate here without modification. In our simulations instead, we opted for the more expensive full pair-wise summations, i.e. the electrostatic interaction of every atom with every other atom in the rod was computed using Coulomb's law. As shown in Figure S2 the bending predicted by the three methods mentioned is significantly different, with the full pair-wise summation being the correct one, as no assumptions are made to speed up the calculations. The reason for the error for the typical Ewald approach is that it assumes that there is no net dipole moment within the cell, which is not the case for the bilayer strip where the dipole varies with degree of bending. Explicit correction for this would therefore be needed. In the case of the Wolf summation, there is an underlying assumption of uniform screening of charge interactions, which does not hold for a finite nanocrystal and therefore again leads to errors. In order to optimize the computing time with LAMMPS we removed any periodic boundary conditions in the simulations and set the interactions cutoff to a value larger than the rod length. Because every atom interacts with all the atoms in the system it is also not necessary to update the neighbors' list, which can also provide a small performance improvement. The calculations with the full pair-wise electrostatics are however at least 20 times slower than with the Ewald summation and a short real space cutoff. For the calculations with the Ewald summation and DSF methods reported in the SI we used a real space cutoff of $10 \AA$, both for the electrostatic and the van der Waals interactions.

\section{ASSOCIATED CONTENT}

\section{Supporting Information.}

Table S1: Force field parameters.

Table S2: Partial atomic charges and mass for iodoform and bromoform. 
Table S3: Crystallographic Information File (CIF) for $P 6_{3}$ iodoform.

Table S4: Crystallographic Information File (CIF) for $\mathrm{P6}_{3}$ bromoform.

Table S5: Lattice parameters comparisons between experimental crystal structure and calculated crystal structure using the force field.

Figure S1: Detailed description of (0001) interface and (10̄0) interface for bimetallic strip analog.

Figure S2: Comparison of electrostatic forces.

Figure S3: Twisted iodoform nanocrystals with diastereomorphous screw dislocations teared along [2$\overline{1} \overline{1} 0]$. (Burgers vector $\vec{b}=\mathrm{c}<0001>$ ).

\section{AUTHOR INFORMATION}

\section{Notes}

The authors declare no competing financial interest.

\section{ACKNOWLEDGMENT}

This work was primarily supported by the Materials Research Science and Engineering Center (MRSEC) program of the National Science Foundation under award number DMR-1420073. We also acknowledge support from DMR-1608374. PR and ALR acknowledge the ARC for funding (DP 140101776, FT 130100463 and DP160100677) and the Pawsey Supercomputer Centre for the provision of computer time.

\section{REFERENCES}

(1) Shtukenberg, A. G.; Gunn, E.; Gazzano, M.; Freudenthal, J.; Camp, E.; Sours, R.; Rosseeva, E.; Kahr, B. Bernauer's bands. ChemPhysChem, 2011, 12, 1558-1571.

(2) Shtukenberg, A. G.; Punin, Y. O.; Gujral, A.; Kahr, B. Growth actuated bending and twisting of single crystals. Angew. Chem. Int. Ed. 2014, 53, 672-699.

(3) Cui, X.; Shtukenberg, A. G.; Freudenthal, J.; Nichols, S. M.; Kahr, B. Circular birefringence of banded spherulites. J. Am. Chem. Soc. 2014, 136, 5481-5490.

(4) Shtukenberg, A. G.; Gujral, A.; Rosseeva, E.; Cui, X.; Kahr, B. Mechanics of twisted hippuric acid crystals untwisting as they grow. CrystEngComm. 2015, 17, 8817-8824.

(5) Zhu, Q.; Shtukenberg, A. G.; Carter, D. J.; Yu, T.; Yang, J.; Chen, M.; Paiteri, P.; Oganov, A. R.; Pokroy, B.; Polishchuk, I.; Bygrave, P. J.; Day, G. M.; Rohl, A. L.; Tuckerman, M. E.; Kahr, B. Resorcinol crystallization from the melt: a new ambient phase and new "riddles". J. Am. Chem. Soc. 2016, 138, 4881-4889.

(6) Shtukenberg, A. G.; Freundenthal, J.; Gunn, E.; Yu, L.; Kahr, B. Glass-crystal growth mode for testosterone propionate. Cryst. Growth Des. 2011, 11, 4458-4462. 
(7) Cui, X.; Nichols, S. M.; Arteaga, O.; Freudenthal, J.; Paula, F.; Shtukenberg, A. G.; Kahr, B. Dichroism in helicoidal crystals. J. Am. Chem. Soc. 2016, 138, 12211-12218.

(8) Cui, X.; Rohl. A. L.; Shtukenberg, A. G.; Kahr, B. Twisted aspirin crystals. J. Am. Chem. Soc. 2013, $135,3395-3398$.

(9) Yang, J.; Hu, C. T.; Shtukenberg, A. G.; Yin, Q.; Kahr, B. L-Malic acid crystallization: polymorphism, semi-spherulites, and polarity, CrystEngComm, 2018, 20, 1383-1289.

(10) Yang, J.; Hu, C. T.; Zhu, X.; Ward, M. D.; Kahr, B. DDT polymorphism and the lethality of crystal forms. Angew. Chem. Int. Ed. 2017, 56, 10165-10169.

(11) Nath, N. K.; Panda, M. K.; Sahoo, S. C.; Naumov, P. Thermally induced and photoinduced mechanical effects in molecular single crystals: A Revival. CrystEngComm, 2014, 16, 1850-1858.

(12) Naumov, P.; Chizhik, S.; Panda, M. K.; Nath, N. K.; Boldyreva, E. Mechanically responsive molecular crystals. Chem. Rev. 2015, 115, 12440-12490.

(13) Abakumov, G. A.; Nevodchikov, V. I. Thermomechanical and photomechanical effects in the crystals of complexes with free radicals. Dokl. Phys. Chem. 1982, 266, 1407-1410.

(14) Boldyreva, E. V.; Sidelnikov, A. A.; Chupakhin, A. P.; Lyakhov, N. Z.; Boldyrev, V. V. Deformation and mechanical fragmentation of the crystals $\left[\mathrm{Co}\left(\mathrm{NH}_{3}\right)_{5} \mathrm{NO}_{2}\right] \mathrm{X}_{2}\left(\mathrm{X}=\mathrm{Cl}, \mathrm{Br}, \mathrm{NO}_{3}\right)$ in the course of linkage photoisomerization. Dokl. Phys. Chem. 1984, 277, 893-896.

(15) Ivanov, F. I.; Urban, N. A. Mechanism of photomechanical deformation of $\beta$-lead azide whisker crystals. React. Solids 1986, 1, 165-170.

(16) Koshima, H.; Ojima, N.; Uchimoto, H. Mechanical motion of azobenzene crystals upon photoirradiation. J. Am. Chem. Soc. 2009, 131, 6890-6891.

(17) Al-Kaysi, R. O.; Müller, A. M.; Bardeen, C. J. Photochemically driven shape changes of crystalline organic nanorods. J. Am. Chem. Soc. 2006, 128, 15938-15939.

(18) Chizhik, S.; Sidelnikov, A.; Zakharov, B.; Naumov, P.; Boldyreva, E. Quantification of photoinduced bending of dynamic molecular crystals: From macroscopic strain to kinetic constants and activation energies, Chem. Sci. 2018, 9, 2319-2335.

(19) Panda, M. K.; Ghosh, S.; Yasuda, N.; Moriwaki, T.; Mukherijee, G. D.; Reddy, C. M.; Naumov, P. Spatially resolved analysis of short-range structure perturbations in a plastically bent molecular crystals. Nat. Chem. 2014, 7, 65-72.

(20) Reddy, C. M.; Krishna, G. R.; Ghosh, S. Mechanical properties of molecular crystals-applications to crystal engineering. CrystEngComm. 2010, 12, 2296-2314.

(21) Subhankar, S.; Desiraju, G. R. Crystal engineering of hand-twisted helical crystals, J. Am. Chem. Soc. 2017, 139, 1975-1983.

(22) Alimi, L. O.; Lama, R.; Smith, V. J.; Barbour, L. J. Hand-twistable plastically deformable crystals of a rigid small organic molecule, Chem. Commun. 2018, 24, 2994-2997.

(23) Krishna, G. R.; Devarapalli, R.; Lai, G.; Reddy, C. M. Mechanically flexible organic crystals achieved by introducing weak interactions in structure: Supramolecular shape synthons, J. Am. Chem. Soc. 2016, 138, 13561-13567. 
(24) Worthy, A.; Grosjean, A.; Pfunder, M. C.; Xu, Y.; Yan, C.; Edwards, G.; Clegg, J. K.; McMurtrie, J. C. Atomic resolution of structural changes in elastic crystals of copper(II) acetylacetonate, Nat. Chem. 2018, 10, 65-69.

(25) Kobatake, S.; Takami, S.; Muto, H.; Ishikawa, T.; Irie, M. Rapid and reversible shape changes of molecular crystals on photo- irradiation. Nature, 2007, 446, 778-781.

(26) Oda, R.; Artzner, F.; Laguerre, M.; Huc, I. Molecular structure of self-assembled chiral nanoribbons and nanotubules revealed in the hydrated state, J. Am. Chem. Soc., 2008, 130 (44), pp $14705-14712$

(27) Tidhar, Y.; Weissman, H.; Tworowski, D.; Rybtchinski, B. Mechanism of crystalline self-assembly in aqueous medium: A combined cryo-TEM/kinetic study, Chem. Euro. J. 2014, 20, 10332 - 10342.

(28) Ponder, J. W.; Case, D. A. Force fields for protein simulations. Adv Protein Chem. 2003, 66, 27-85.

(29) Cheatham, T. E. $3^{\text {rd }}$; Kollman, P. A. Molecular dynamics simulations of nucleic Acids. Annu Rev Phys Chem. 2000, 51, 435-471.

(30) Wang, J.; Wolf, R. M.; Caldwell, J. W.; Kollman, P. A.; Case, D. A. Development and testing of a general amber force field. J. Comput. Chem. 2004, 25, 1157-1174.

(31) Nemkevich, A.; Bürgi, H.B.; Spackman, M. A.; Corry, B. Molecular dynamics simulations of structure and dynamics of organic molecule crystals. Phys. Chem. Chem. Phys. 2010, 12, 1491614929.

(32) Boyd, N. J.; Wilson, M. R. Optimization of the GAFF force field to describe liquid crystal molecules: the path to a dramatic improvement in transition temperature predictions. Phys. Chem. Chem. Phys. 2015, 17, 24851-24865.

(33) Myers, R.; Torrie, B. H.; Powell, B. M. Crystal structures of solid bromoform. J. Chem. Phys. 1983, 79, 1495-1504.

(34) Wykoff, R. W. G. Crystal Structures, vol. 5, Interscience, New York, 1964.

(35) Kitaigorodskii, A. I.; Khostsyanova, T.L.; Struchkov, Y.T. Crystal structure of iodoform. Dokl. Akad. Nauk SSSR. 1951, 78, 1161.

(36) Iwata, Y.; Watanabe T. Reinvestigation of the crystal structure of iodoform by neutron diffraction, Ann. Rep. Res. React. Inst. Kyoto U. 1979, 7, 87-93.

(37) Bertolotti, F.; Curetti, N.; Benna, P.; Gervaso, G. The effects of P-T changes on intermolecular interactions in crystal structure of iodoform. J. Mol. Struct. 2013, 1041, 106-112.

(38) Timoshenko, S. Analysis of bi-metal thermostats. J. Opt. Soc. Am. 1925, 11, 233-255.

(39) Sahoo, S. C.; Sinha, S. B.; Kiran, M. S. R. N.; Ramamurty, U.; Dericioglu, A. F.; Reddy, C. M.; Naumov, P. Kinematic and mechanical profile of the self-actuation of thermosalient crystal twins of 1,2,4,5-tetrabromobenzene: A molecular crystalline analogue of a bimetallic strip. J. Am. Chem. Soc. 2013, 135, 13843-13850.

(40) Cahn, J. W.; Hanneman, R. E. (111) surface tension of III-V compounds and their relationship to spontaneous bending of thin crystals. Surf. Sci. 1964, 1, 387-398.

(41) Zang, J.; Huang, M.; Liu, F. Mechanism for nanotube formation from self-bending nanofilms driven by atomic-scale surface stress imbalance. Phys. Rev. Lett. 2007, 98, 146102.

(42) Zang, J. and Liu, F. Modified Timoshenko formula for bending of ultrathin strained bilayer films. Appl. Phys. Lett. 2008, 92, 021905. 
(43) Webb, W. W.; Forgeng, W. D. Growth and defect structure of sapphire microcrystals. J. Appl. Phys. 1957, 28, 1449-1454.

(44) Webb, W. W.; Dragsdorf, R. D.; Forgeng, W. D. Dislocations in whiskers. Phys. Rev. 1957, 108, 498-499.

(45) Eshelby, J. D. Screw dislocations in thin rods. J. Appl. Phys. 1953, 24, 176-179.

(46) Eshelby, J. D. The twist in a crystal whisker containing a dislocation. Philos. Mag. 1958, 3, 440447.

(47) Bierman, M. J.; Lau, Y. K. A.; Kvit, A. V.; Schmitt, A. L.; Jin, S. Dislocation-driven nanowire growth and Eshelby twist. Science. 2008, 320, 1060-1063.

(48) Zhu, J.; Peng, H.; Marshall, A. F.; Barnett, D. M.; Nix, W. D.; Cui, Y. Formation of chiral branched nanowires by the Eshelby twist. Nat. Nanotechnol. 2008, 3, 477-481.

(49) Nikiforov, I.; Zhang, D. B.; Dumitrică, T. Screw dislocations in $<100>$ silicon nanowires: an objective molecular dynamics study. J. Phys. Chem. Lett. 2011, 2, 2544-2548.

(50) Walker, A. M.; Slater, B.; Gale, J. D.; Wright, K. Predicting the structure of screw dislocations in nanoporous materials, Nature Materials, 2004, 3, 715-720.

(51) Walker, A. M.; Gale, J. D.; Slater, B.; Wright, K. Atomic scale modelling of the cores of dislocations in complex materials part 1: methodology, Phys. Chem. Chem. Phys. 2005, 7, 32273234.

(52) Walker, A. M.; Gale, J. D.; Slater, B.; Wright, K. Atomic scale modelling of the cores of dislocations in complex materials part 2: applications, Phys. Chem. Chem. Phys. 2005, 7, 32353242 .

(53) Levitt, L. S. R and S screw dislocations: The chiral growth of crystals of chiral molecules. Indian J. Phys. 1975, 49, 696-697.

(54) Shtukenberg, A. G.; Poloni, L. N.l; Zhu, Z.; An, Z.; Bhandari, M.; Song, P.; Rohl, A. L.; Kahr, B.; Ward, M. D. Dislocation-actuated growth and inhibition of hexagonal $L$-cystine crystallization at the molecular level. Cryst. Growth Des. 2015, 15, 921-934.

(55) Stoica, C.; van Enckevort, W. J. P.; Meekes, H.; Vlieg, E. Interlaced spiral growth and step splitting on a steroid crystal. J. Cryst Growth. 2007, 299, 322-329.

(56) Kitagawa, D.; Tsujioka, H.; Tong, F.; Dong, X.; Bardeen, C. J.; Kobatake, S. Control of photomechanical crystal twisting by illumination direction, J. Am. Chem. Soc. 2018, 140, 42084212.

(57) Samanta, R.; Ghosh, S.; Devarapalli, R.; Reddy, C. M. Visible light mediated photopolymerization in single crystals: Photomechanical bending and thermomechanical unbending, Chem. Mater. 2018, 30, 577-581.

(58) Gupta, P.; Karothu, D. P.; Ahmed, E.; Naumov, P. Thermally twistable, photobendable, elastically deformable self-healable soft crystals, Angew. Chem. Int. Ed. 2018, 230, 8634-8638.

(59) Rai, R.; Krishnan, B. P.; Sureshan, K. M. Chirality-controlled spontaneous twisting of crystals due to thermal topochemical reaction, Proc. Natl. Acad. Sci. 2018, 171896511

(60) Tong, F.; Hanson, M. P.; Bardeen, C. J. Analysis of reaction kinetics in the photomechanical molecular crystal 9-methyulanthracene using an extended Finke-Watzky model, Phys. Chem. Chem. Phys. 2016, 18, 31936-31945. 
(61) Nath, N. K.; Pejov, L.; Nichols, S. M.; Hu, C.; Saleh, N.; Kahr, B.; Naumov, P. Model for photoinduced bending of slender molecular crystals, J. Am. Chem. Soc. 2014, 136, 2757-2766.

(62) Plimpton, S. Fast parallel algorithms for short-range molecular dynamics. J. Comput. Phys. 1995, 117, 1-19.

(63) Kendall, R.A.; Apra, E.; Bernholdt, D.E.; Bylaska, E.J.; Dupuis, M.; Fann, G.I.; Harrison, R.J.; Ju, J.; Nichols, J.A.; Nieplocha, J.; Straatsma, T.P.; Windus, T.L.; Wong, A.T. High performance computational chemistry: an overview of NWChem a distributed parallel application. Comput. Phys. Commun. 2000, 128, 260-283.

(64) Gale, Julian. D.; Rohl, A. L. The general utility lattice program (GULP). Mol Simul. 2003, 29, 291341. 